\title{
ON THE NUMBER OF GENERATORS OF POWERS OF AN IDEAL
}

\author{
JUDITH D. SALLY ${ }^{1}$
}

ABSTRACT. Let $I$ be an ideal of a quasi-local ring. In this note we consider the question of how small-in terms of numbers of generators-the powers of the ideal $I$ can be.

In this note all rings are commutative with identity. If $I$ is an ideal of a ring, $v(I)$ denotes the minimal number, which may be infinite, of generators of $l$.

Proposition 1. Let $I$ be an ideal of a quasi-local ring $A$. If, for some integer $t \geq 1, v\left(I^{t}\right)=1$, then either $v\left(I^{k}\right)=1$ for all positive integers $k$ or $I$ consists of zero divisors. If, for some integer $t>1, v\left(I^{t}\right)=2$, then either $v\left(I^{k}\right)=2$ for all positive integers $k$ or $I$ consists of zera divisors.

We will use the following lemmas in the proof of the proposition.

Lemma 2. Let $I$ be an ideal of a ring $R$. Let $J$ and $K$ be subsets of $I$ such that $J \subseteq I$ and $K \subseteq I^{t-1}$ for some positive integer $t$. If $I^{t}=J K R$, then $I^{t+1}=J^{2} K R$.

Proof. $I^{t+1}=I J K R=J I K R \subseteq J I^{t}=J^{2} K R$.

It is an immediate consequence of Lemma 2 that if some power $I^{t}$ of an ideal $I$ is finitely generated then so is every higher power. Since $I^{t}$ is generated by sums of monomials of degree $t$ in elements of $I$, take $J$ to be the ideal generated by all the elements of $I$ which appear in the monomials in such a generating set. Then $J$ is a finitely generated ideal contained in $I, I^{t}=J^{t}$ and, by Lemma $2, I^{t^{+k}}=J^{t^{+} k}$ for $k \geq 0$.

If an element $x$ in $I$ appears in $j$ monomials in some minimal generating set for $I^{t}$ we will say that $I^{t}$ has an overlap of length $j$. It follows from Lemma 2 that if $v\left(I^{t}\right)=n$ and if $I^{t}$ has an overlap of length $n$, then $v\left(I^{t^{+} k}\right)$ $\leq n$ for $k \geq 0$. (In particular, if an ideal $I$ in a quasi-local ring has some power principal, then every higher power is principal.)

If $(A, \mathbf{m})$ is a quasi-local ring and $u$ an indeterminate we will often make use of the faithfully flat change of rings $A \rightarrow A(u)=A[u]_{\mathrm{m} A[u]}$.

Received by the editors September 13, 1974.

AMS (MOS) subject classifications (1970). Primary 13C05, 13G05, $13 \mathrm{H} 99$.

Key words and phrases. Quasi-local ring, ideal, faithfully flat ring extension.

1 The author received partial support from the National Science Foundation. 
Lemma 3. Let $(A, \mathrm{~m})$ be a quasi-local ring and I a finitely generated ideal. Let $t$ be any integer $>1$. Then either $I^{t}$ is principal or $I^{t} A(u)$ has an overlap of length 2.

Proof. Let $I=\left(x_{1}, \ldots, x_{n}\right)$ and suppose that $v\left(I^{t}\right)>1$, say

$$
I^{t}=\left(x_{i_{1}}^{\alpha_{1}} \ldots x_{i_{r}}^{\alpha_{r}}, \ldots, x_{j_{1}}^{\beta_{1}} \ldots x_{j_{s}}^{\beta_{s}}\right)
$$

with $\alpha_{i}, \beta_{i}$ positive integers such that $\alpha_{1}+\cdots+\alpha_{r}=\beta_{1}+\cdots+\beta_{s}=t$. Assume that every $x_{i}$ appears in at most one monomial in this generating set for $I^{t}$. Now

$$
x_{i_{1}} x_{j_{1}}^{\beta_{1}-1} x_{j_{2}}^{\beta_{2}} \cdots x_{j_{s}}^{\beta_{s}}=a x_{i_{1}}^{\alpha_{1}} \cdots x_{i_{r}}^{\alpha_{r}}+\cdots+b x_{j_{1}}^{\beta_{1}} \cdots x_{j_{s}}^{\beta_{s}}
$$

for some $a, \ldots, b \in A$. If one of the coefficients $a, \ldots, b$ is not in $\mathrm{m}$ we can replace the generator it multiplies by $x_{i} x_{j_{1}}^{\beta_{1}-1} x_{j_{2}}^{\beta_{2}} \ldots x_{j}^{\beta_{s}}$ and produce an overlap in $I^{t}$ itself. So we assume that all the coefficients are in $\mathrm{m}$. We pass to the ring $A(u)$ and change the basis of $I A(u)$ as follows:

$$
x_{i_{1}}=y_{i_{1}}+u y_{j_{i}} ; \quad x_{k}=y_{k} \text { for } k \neq i_{1} .
$$

Then

$$
I A(u)=\left(y_{1}, \ldots, y_{n}\right) \text { and } I^{t} A(u)=\left(y_{i_{1}}^{a_{1}} \ldots y_{i_{r}}^{a_{r}}, \ldots, y_{j_{1}}^{\beta_{1}} \ldots y_{j_{s}}^{\beta_{s}}\right) A(u) \text {, }
$$

for

$$
I^{t} \subseteq\left(y_{i_{1}}^{\alpha_{1}} \cdots y_{i_{r}}^{a_{r}}, \ldots, y_{j_{1}}^{\beta_{1}} \cdots y_{j_{s}}^{\beta_{s}}\right) A[u]+u I^{t} A[u]
$$

Now $(*)$ gives

$$
y_{i_{1}} y_{j_{1}}^{\beta_{1}-1} y_{j_{2}}^{\beta_{2}} \cdots y_{j_{s}}^{\beta_{s}}+(u-b) y_{j_{1}}^{\beta_{1}} \cdots y_{j_{s}}^{\beta_{s}} \in \mathbf{m} I^{t} A(u) .
$$

Hence, if we substitute $y_{i_{1}} y_{j_{1}}^{\beta_{1}-1} y_{j_{2}}^{\beta_{2}} \cdots y_{j_{s}}^{\beta_{s}}$ for the generator $y_{j_{1}}^{\beta_{1}} \cdots y_{j_{s}}^{\beta_{s}}$ of $I^{t} A(u)$, we have that $I^{t} A(u)$ has an overlap of length 2 .

Corollary. Let $A$ be a quasi-local ring and I an ideal suci' that for some $t>1, v\left(I^{t}\right)=2$. Then $v\left(I^{t^{+}}\right) \leq 2$ for all $k \geq 0$.

Proof. By Lemma 2 we may assume that $I$ is finitely generated. $I^{t} A(u)$ has 2 generators and, by Lemma 3, an overlap of length 2 . Hence $v\left(I^{t+k} A(u)\right)$ $\leq 2$ and $v\left(I^{t+k}\right) \leq 2$.

Proof of Proposition 1. If $I$ is not principal, let $t>1$ be the least integer such that $v^{t}\left(I^{t}\right)=1$. Say $I^{t}=\left(x_{1}^{\alpha} \ldots x_{r}^{\alpha} r\right)$, where the $\alpha_{i}$ are positive integers such that $\alpha_{1}+\cdots+a_{r}=t$ and the $x_{i}$ are in $I$. Since it is enough to show that some $x_{i}$ is a zero divisor we may assume that $I=\left(x_{1}, \ldots, x_{r}\right)$ and that $x_{1}^{a_{1}-1} x_{2}^{a_{2}} \ldots x_{r}^{a_{r}}$ is one of the generators in a minimal basis for $I^{t-1}$. Let $z$ be another such generator. Then the relation $x_{1} z=b x_{1}{ }_{1} \ldots$ 
$x_{r}^{a}$, for some $b$ in $A$, shows that $x_{1}$ is a zero divisor. To prove the second statement, suppose that $t>1$ is a positive integer such that $v\left(I^{t}\right)=2$ and $v\left(I^{t-1}\right)>2$. By passing to the ring $A(u)$ we may assume that $I^{t}$ has a minimal generating set of the form $x_{1} x_{2} \alpha_{2} \ldots x_{r}^{a_{r}}, x_{1} x_{r+1}^{a_{r+1}} \ldots x_{s}^{a_{s}}$ with $x_{1}, \ldots$ $x_{s} \in I$ and $\alpha_{i}$ positive integers such that $a_{1}+\cdots+a_{r}=a_{r+1}+\cdots+\alpha_{s}=t$ - 1 . Then there is a $z$ in $I^{t-1} \backslash\left(x_{2}^{a_{2}} \ldots x_{r}^{a_{r}}, x_{r+1}^{a_{r+1}} \ldots x_{s}^{a_{s}}\right)$. The relation

$$
z x_{1}=a x_{1} x_{2}^{a_{2}} \cdots x_{r}^{a_{r}}+b x_{1} x_{r+1}^{a}{ }_{r+1} \ldots x_{s}^{a}{ }_{s} \text {, }
$$

for some $a, b$ in $A$, shows that $x_{1}$ is a zero divisor and that $I A(u)$ consists of zero divisors. Hence (cf. [3]) $l$ consists of zero divisors.

The following example was shown to me by P. Eakin and W. Heinzer. (The reference [1] was supplied by the referee.) The example demonstrates that "very big" ideals in quasi-local domains can have powers generated by 3 elements. Let $n \in\{1,2, \ldots, \infty\}$. Let $S$ be any ring having an ideal $J$ minimally generated by $n$ elements. Let $R=S\left[\left[x^{4}, x^{5}, J x^{11}\right]\right]$, where $x$ is an indeterminate, and consider the ideal $I=\left(x^{4}, x^{5}, J x^{11}\right) R . \quad v(I)=n+2$, but $v\left(I^{2}\right)=3$ since $I^{2}=\left(x^{8}, x^{9}, x^{10}\right) R$. Variation of the semigroup used as exponents for $x$ leads to many such examples. Notice, however, that the ciomain $R$ has dimension greater than one. What happens in the one-dimensional local case, except for some very special ideals (e.g. [2], [4]), is an open question. It is possible that the following generalization of Lemma 3 hcids: if $I$ is an ideal of a quasi-local ring $A$ with $v\left(I^{t}\right)=k$ where $t \geq k$, then $v\left(I^{t^{+}} j\right) \leq k$ for all $j \geq 0$. * The combinatorial methods used above give a rather lengthy verification of this for $k=3$, but this does not seem to be a good way to attack the general case.

* Added in proof. This conjecture has been proved by Eakin and Sathaye, Prestable ideals, J. Algebra (to appear). Also pertinent are the papers A note on the Hilbert function of a one-dimensional Cohen-Macaulay ring (preprint) by Herzog and Waldi, and Hilbert functions of graded algebras (preprint) by Stanley.

\section{REFERENCES}

1. R. Gilmer, On factorization into prime ideals, Comment. Math. Helv. 47 (1972), 70-74. MR $46 \# 5310$.

2. E. Matlis, The multiplicity and reduction number of a one-dimensional local ring, Proc. London Math. Soc. (3) 26 (1973), 273-288. MR $47 \# 1802$.

3. N. H. McCoy, Rings and ideals, Carus Math. Monographs, no. 8, Open Court, LaSalle, Ill., 1948. MR 10, 96.

4. J. Sally and W. Vasconcelos, Stable rings, J. Pure Appl. Algebra 4 (1974), $319-336$.

DEPARTMENT OF MATHEMATICS, NORTHWESTERN UNIVERSITY, EVANSTON, ILLINOIS 60201 